CORRIGENDUM

doi:10.1038/nature07133

\title{
Neurophysiology: Sensing temperature without ion channels
}

Brandon R. Brown

\section{Nature 421, 495 (2003)}

My Brief Communication about thermoelectricity in shark gels neglected a systematic effect of surface electrochemistry: electrode potentials vary with temperature in electrolyte solutions. However, silver leads in sea water ${ }^{1}$ and accepted values for likely electrode reactions ${ }^{2}$ show a sign opposing the gel signals, making it unlikely that an artefactual signal is the origin. Our subsequent work ${ }^{3}$ discussed artefacts and repeated the signal with platinum electrodes. Although another report ${ }^{4}$ finds a zero signal using salt bridges, it ignores thermopower in gel-filled leads, which risks building a 'null thermocouple' from two similar materials (see ref. 5, for example). A temperature function of the electrosensors is not known, but the thermoelectric transduction hypothesis still stands.

1. Sanford, T. B. Measurements and Interpretations of Motional Electric Fields in the Sea. $\mathrm{PhD}$ thesis, Massachusetts Institute of Technology (1967).

2. Milazzo, G. \& Caroli, S. Tables of Standard Electrochemical Potentials (John Wiley and Sons, New York, 1978).

3. Brown, B. R., Hughes, M. E. \& Russo, C. Thermoelectricity in natural and synthetic hydrogels. Phys. Rev. E 70, 031917 (2004).

4. Fields, R. D., Fields, K. D. \& Fields, M. C. Semiconductor gel in shark sense organs? Neurosci. Lett. 426, 166-170 (2007).

5. Kasap, S. O. Principles of Electronic Materials and Devices 278-284 (McGraw Hill, San Francisco, 2000).

doi:10.1038/nature07134

An extremely luminous $X$-ray outburst at the birth of a supernova

A. M. Soderberg, E. Berger, K. L. Page, P. Schady, J. Parrent, D. Pooley, X.-Y. Wang, E. O. Ofek, A. Cucchiara, A. Rau, E. Waxman, J. D. Simon, D. C.-J. Bock, P. A. Milne, M. J. Page, J. C. Barentine, S. D. Barthelmy, A. P. Beardmore, M. F. Bietenholz, P. Brown, A. Burrows,

D. N. Burrows, G. Bryngelson, S. B. Cenko, P. Chandra, J. R. Cummings, D. B. Fox, A. Gal-Yam, N. Gehrels, S. Immler, M. Kasliwal, A. K. H. Kong, H. A. Krimm, S. R. Kulkarni, T. J. Maccarone, P. Mészáros, E. Nakar, P. T. O'Brien, R. A. Overzier, M. de Pasquale, J. Racusin, N. Rea \& D. G. York

Nature 453, 469-474 (2008)

In this Article, the surname of co-author G. Bryngelson was misspelled as G. Byrngelson. 